\title{
Espécies de Canoparmelia s.l. (Parmeliaceae, ascomicetes liquenizados) da região tocantina, MA e TO, Brasil ${ }^{1}$
}

\author{
Iane Paula Rego Cunha ${ }^{2,4}$, Marcelo Pinto Marcelli ${ }^{3}$ e Eugênia Cristina Pereira ${ }^{2}$
}

Recebido: 23.12.2014; aceito: 11.02.2015

\begin{abstract}
Canoparmelia species s.l. (Parmeliaceae, lichenized ascomycetes) of the tocantinan region, Maranhão and Tocantins States, Brazil). Canoparmelia amazonica, C. [Crespoa] crozalsiana, C. cryptochlorophaea, C. [Parmelinella] salacinifera, and C. texana are registered for the Brazilian states of Maranhão and/or Tocantins. All these are new records in the region, except for C. salacinifera, already known for Maranhão State. All species are commented and C. martinicana, a new record for Brazil (Maranhão State), is described in detail and illustrated.
\end{abstract}

Keywords: cerrado vegetation, lichens, Maranhão, Tocantins

RESUMO - (Espécies de Canoparmelia s.l. (Parmeliaceae, ascomicetes liquenizados) da região tocantina, MA e TO, Brasil). Canoparmelia amazonica, C. [Crespoa] crozalsiana, C. cryptochlorophaea, C. [Parmelinella] salacinifera e C. texana, são mencionadas para os Estados do Maranhão e/ou Tocantins, Brasil. Todas são novas ocorrências para a região, exceto C. salacinifera, já conhecida do Estado do Maranhão. São apresentados comentários para todas as espécies e C. martinicana, uma nova citação para o Brasil (Estado do Maranhão), é detalhadamente descrita e ilustrada.

Palavras-chave: cerrado, liquens, Maranhão, Tocantins

\section{Introdução}

Canoparmelia é um gênero cosmopolita, com centro de especiação nas Américas e no continente Africano. Caracteriza-se pelo talo folioso adnato ao substrato, com rizinas simples que chegam até as margens dos lobos ou lacínios; geralmente apresenta cor cinza claro ou cinza esverdeado (atranorina presente no córtex superior), ou raramente amarelo esverdeado (ácido úsnico presente no córtex superior); os lobos ou lacínios são frequentemente maculados, eciliados, com face inferior de castanho a negra, e pequenos em relação à maioria dos outros gêneros de Parmeliaceae (Elix et al. 1986, Elix 1994).

Recentemente, as espécies de Canoparmelia cujo talo é tipicamente foveolado foram segregadas no novo gênero Crespoa (Lendemer \& Hodkinson 2012). Por outro lado, as espécies com ácido salazínico medular e cílios restritos às axilas, foram recentemente recombinadas no gênero Parmelinella (Benatti 2012, 2014). Por essa razão, esses gêneros foram aqui reunidos como Canoparmelia s.l.

Exceto pela citação de Parmelinella salacinifera (Hale) Marcelli \& Benatti para o Estado do Maranhão (Hale 1976, como Pseudoparmelia salacinifera (Hale) Hale), não há citações de espécies de Canoparmelia para os Estados do Maranhão e Tocantins.

Alguns municípios do sul do Estado do Pará, sudoeste do Estado do Maranhão e nordeste do Estado do Tocantins formam a região tocantina, onde este trabalho foi realizado. Trata-se de uma área não formalmente reconhecida e de limites um tanto variáveis, de acordo com os autores ou os interesses econômicos, cuja altitude está mais comumente entre 80 e $200 \mathrm{~m}$, coberta principalmente por vegetação de cerrado, mas também com matas de transição

1. Parte da Tese de Doutorado da primeira Autora, Programa de Pós-Graduação em Biologia Vegetal da Universidade Federal de Pernambuco

2. Universidade Federal de Pernambuco, Centro de Filosofia e Ciências Humanas, Departamento de Ciências Geográficas, 50740-901 Recife, PE, Brasil

3. Instituto de Botânica, Núcleo de Pesquisas em Micologia, Av. Miguel Stéfano 3687, Água Funda, CEP 04301-902 São Paulo, SP, Brasil

4. Autor para correspondência: mpmarcelli@msn.com 
(pré-amazônia) e floresta amazônica; o clima é definido como tropical semi-úmido, com médias de temperatura variando entre 25 e $29^{\circ} \mathrm{C}$ (Sousa 2009, Martins \& Oliveira 2011, TOCANTINS 2011); e estudos de levantamento da micota liquênica são inexistentes.

Este trabalho é parte da tese de doutorado da primeira Autora, que realizou o levantamento das espécies de Parmeliaceae da região tocantina e citou as seis espécies de Canoparmelia encontradas nos Estados do Maranhão e Tocantins; descreveu em detalhe C. martinicana (Nyl.) Elix \& Hale, citada pela primeira vez no Brasil e para a qual os dados de literatura são escassos e as duas descrições existentes (Hale 1976, Nylander 1885) são por demais simples e insuficientes para uma identificação precisa.

\section{Material e métodos}

Embora algumas amostras tenham sido obtidas sobre árvores de área urbana, a grande maioria das coletas foi realizada em deslocamentos ao acaso em áreas de vegetação natural constituída principalmente por cerrados, com procura ativa de espécies de Parmeliaceae na área de 13 municípios selecionados pelo fato de ainda conterem áreas naturais suficientemente preservadas para portarem líquens (tabela 1). Todo tipo de substrato em todo hábitat e micro-hábitat encontrado foi investigado.

Os procedimentos gerais de coleta seguiram Hale (1979), Brodo et al. (2001) e Canêz \& Marcelli (2006).

As análises morfológicas foram realizadas sob microscópios estereoscópico e óptico, seguindo o protocolo básico para descrição de espécimes de Parmeliaceae (Canêz \& Marcelli, 2006), desenvolvido pelo Grupo de Estudos Liquenológicos (GEL) do Instituto de Botânica (São Paulo) e que tem sido constantemente atualizado, atualmente exigindo a análise de mais de 150 características, inclusive aquelas aqui incluídas na descrição de Canoparmelia martinicana. Várias das características utilizadas, como por exemplo, o acetinado ou aveludado das superfícies, e outras das tradicionalmente utilizadas e cujos conceitos variam de autor para autor se tornaram estritamente definidas, como lobos, lacínios, lóbulos, lacínulos, rizinas e cílios para possibilitar a descrição minuciosa de detalhes importantes na distinção de espécies brasileiras de Parmeliaceae (B.R. Hora, dados não publicados).

Para as análises químicas foram utilizados testes de coloração, cromatografia em camada delgada
(CCD) e fluorescência sob luz ultravioleta, segundo a metodologia de Walker \& James (1980), Huneck \& Yoshimura (1996), Orange et al. (2001), Canêz \& Marcelli (2006) e Elix (2014).

O material examinado foi depositado no Herbário Maria Eneyda P. Kauffmann Fidalgo (SP) do Instituto de Botânica, em São Paulo, SP, Brasil.

\section{Resultados e Discussão}

A investigação das zonas urbanas e dos hábitats e substratos naturais da região tocantina revelou a presença de seis espécies conhecidas de Canoparmelia s.l., uma delas citada pela primeira vez para o Brasil (C. martinicana) e quatro encontradas pela primeira vez na região tocantina. Apenas $C$. salacinifera já havia sido citada para o Estado do Maranhão, porém fora da região tocantina (Hale, 1976). Excetuando as poucas amostras obtidas em troncos de árvores de zona urbana, todas foram coletadas sobre árvores de cerrado em seis dos 13 municípios incluídos na amostragem (tabela 1).

As espécies são apresentadas abaixo em ordem alfabética e suas principais características comentadas, exceto C. martinicana, cuja escassez de informação na literatura tornou importante a apresentação de uma descrição detalhada.

As descrições das espécies encontradas na literatura são por demais simples e resumidas, não permitindo comparações entre si e com nossos espécimes. As únicas exceções são as descrições fornecidas nas dissertações de Jungbluth (2006) e Zanetti (2013) para espécimes dos cerrados do Estado de São Paulo; nesse caso as diferenças, quando existentes, são comentadas. Porém, na grande maioria dos casos, não houve diferenças morfológica significativas entre o material paulista e o aqui estudado.

Canoparmelia amazonica (Nyl.) Elix \& Hale, Mycotaxon 27: 277. $1986 \equiv$ Parmelia amazonica Nyl., Flora 68: 611. 1885. Tipo: Brasil, Amazonas, Santarém, leg. Spruce 111 (lectotipo: H-Nyl; isolectotipos: BM, G, NY, W, PC), fide Hale (1976).

Distribuição conhecida: pantropical, pantemperado (Hale 1976); no Brasil foi citada para BA, MG. MT, PA, PR, RJ e SP (Jungbluth 2006).

Descrição e ilustração: Hale (1976), Jungbluth (2006).

Testes de coloração: córtex superior $\mathrm{K}+$ amarelo, UV-; medula $\mathrm{K}+$ fraco amarelo, $\mathrm{C}-, \mathrm{KC}-$ ou $\mathrm{KC}+$ rosa, $\mathrm{P}+$ amarelo/laranja, UV-. 
Substâncias de importância taxonômica (CCD): atranorina no córtex superior e ácido protocetrárico na medula.

Material examinado: BRASIL. MARANHão: Município de São João do Paraíso, em árvore viva no cerrado, 7-IX-2009, I.P.R. Cunha, J. Bordin, M.F. Silva \& M.Q. Carneiro 14; Município de Carolina, Fazenda Santa Rita, em árvore viva no cerrado, 22-III-2009, Cunha, I.P.R. \& M.A.L. Dias 21632, 21717, 22245, 24900, 29205, 29206; Município de Governador Edson Lobão, 13-VI-2009, I.P.R. Cunha 10967; Município de São Pedro dos Crentes, em árvore viva no cerrado, 23-VII-2009, I.P.R. Cunha \& M.A.L. Dias 167, 168; idem, 13-X-2009, I.P.R. Cunha, M.P. Marcelli, \& M.A.L. Dias 58A. Tocantins: Município de Itaguatins, Fazenda São Paulo, em tronco de árvore viva, 1-I-2009, I.P.R. Cunha \& M.A.L. Dias 51.

Canoparmelia amazonica é uma espécie muito comum em todo o Brasil, principalmente em locais ensolarados dos cerrados (Jungbluth 2006), como na região estudada. É facilmente reconhecida pelo talo lobado bastante adnato, acinzentado claro a esbranquiçado, não maculado, com isídios laminais, o lado de baixo negro e a reação $\mathrm{P}+$ amarelo forte ou alaranjado na medula, indicando a presença de ácido protocetrárico.

No Brasil, apenas C. martinicana (Nyl.) Elix \& Hale apresenta a combinação de isídios e presença de ácido protocetrárico; entretanto, essa espécie possui a parte terminal dos lobos bastante maculada e evidentemente escrobiculada, além de inúmeras diferenças no morfologia e ontogenia dos isídios, que comumente apresentam o ápice decorticado, com a consequente exposição da medula (vide comentário sob essa espécie).

No Brasil, outras espécies isidiadas são sempre bastante maculadas e os testes de coloração na medula são bastante diferentes devido à presença de outras substâncias de importância taxonômica.

Esta espécie está sendo citada pela primeira vez para os Estados do Maranhão e Tocantins.

Crespoa crozalsiana (B. de Lesd. ex Harm.) Lendemer \& Hodkinson, North American Fungi 7: 3. $2012 \equiv$ Canoparmelia crozalsiana (B. de Lesd.) Elix \& Hale, Mycotaxon 27:278. 1986. EParmelia crozalsiana B. de Lesd., Lichens de France 4: 555. 1910. Tipo: França, Hérault, Agde, leg. De Crozals, May 1909 (lectotipo: US!).

Distribuição conhecida: pantropical, pantemperado (Hale 1976). No Brasil foi citada para MG, RJ, RS e SP (Jungbluth 2006).

Descrição e ilustração: Hale (1976), Jungbluth (2006), Zanetti (2014).

Testes de coloração: córtex superior $\mathrm{K}+$ amarelo, UV-; medula $\mathrm{K}+$ amarelo vivo, $\mathrm{C}-, \mathrm{KC}-, \mathrm{P}+$ salmão, UV-.

Tabela 1. Municípios da região tocantina, MA e TO, Brasil, incluídos na amostragem de espécies de Parmeliaceae, com indicação (*) daqueles onde foram encontrados os espécimes de Canoparmelia s.l. aqui estudados.

Table 1. The tocantinan municipalities, Maranhão and Tocantins States, Brazil, included in the Parmeliaceae sampling, indicating $(*)$ those where the specimens of Canoparmelia s.l. here studied were found.

\begin{tabular}{lccc}
\hline Município & Estado & Latitude (S) & Longitude (W) \\
\hline Açailândia & MA & $4^{\circ} 56^{\prime} 48^{\prime \prime}$ & $47^{\circ} 30^{\prime} 17^{\prime \prime}$ \\
Araguatins & TO & $5^{\circ} 39^{\prime} 04^{\prime \prime}$ & $48^{\circ} 07^{\prime} 28^{\prime \prime}$ \\
Axixá do Tocantins & TO & $5^{\circ} 36^{\prime} 59^{\prime \prime}$ & $47^{\circ} 47^{\prime} 10^{\prime \prime}$ \\
Campestre do Maranhão & MA & $6^{\circ} 10^{\prime} 20^{\prime \prime}$ & $47^{\circ} 21^{\prime} 49^{\prime \prime}$ \\
Carolina * & MA & $7^{\circ} 19^{\prime} 58^{\prime \prime}$ & $47^{\circ} 28^{\prime} 10^{\prime \prime}$ \\
Estreito & MA & $6^{\circ} 33^{\prime} 38^{\prime \prime}$ & $47^{\circ} 27^{\prime} 04^{\prime \prime}$ \\
Governador Edson Lobão * & MA & $5^{\circ} 44^{\prime} 58^{\prime \prime}$ & $47^{\circ} 21^{\prime} 33^{\prime \prime}$ \\
Itaguatins * & TO & $5^{\circ} 46^{\prime} 08^{\prime \prime}$ & $47^{\circ} 29^{\prime} 00^{\prime \prime}$ \\
Porto Franco & MA & $6^{\circ} 20^{\prime} 18^{\prime \prime}$ & $47^{\circ} 23^{\prime} 57^{\prime \prime}$ \\
São Bento do Tocantins * & TO & $6^{\circ} 01^{\prime} 13^{\prime \prime}$ & $47^{\circ} 54^{\prime} 08^{\prime \prime}$ \\
São João do Paraíso * & MA & $6^{\circ} 27^{\prime} 40^{\prime \prime}$ & $47^{\circ} 03^{\prime} 27^{\prime \prime}$ \\
São Pedro dos Crentes * & MA & $6^{\circ} 49^{\prime} 35^{\prime \prime}$ & $46^{\circ} 31^{\prime} 55^{\prime \prime}$ \\
Sítio Novo & MA & $5^{\circ} 36^{\prime} 00^{\prime \prime}$ & $47^{\circ} 38^{\prime} 29^{\prime \prime}$ \\
\hline
\end{tabular}


Substâncias de importância taxonômica (CCD): atranorina no córtex superior; ácidos stíctico, constíctico, criptostíctico e hipoconstíctico na medula.

Material examinado: BRASIL. MARANHÃo: Município de Carolina, Fazenda Santa Rita, em árvore viva, 22-VII-2004, I.P.R. Cunha \& M.A.L. Dias 523.

Crespoa crozalsiana é caracterizada pela superfície superior foveolada associada à presença de sorais laminais orbiculares (podem se fundir quando mais velhos na região proximal do talo) e ácidos do complexo stíctico na medula (reação $\mathrm{K}+$ amarelo vivo).

Crespoa carneopruinata (Zahlbr.) Lendemer \& Hodkinson tem morfologia e química semelhantes, porém apresenta ramificações mais estreitas (lacínios) com até $2,5 \mathrm{~mm}$ de largura máxima, enquanto C. crozalsiana tem ramificações mais largas e ramos (sublacínios e sublobos) com 1,5 a 6,0 mm de largura. A distinção entre essas espécies tem sido tradicionalmente baseada apenas nessa diferença de tamanho e considerada discutível por vários autores que, entretanto, sempre se recusaram a sinonimizá-las (Nash \& Elix 2002, Jungbluth 2006, Zanetti 2014).

Canoparmelia texana (Tuck.) Elix \& Hale e C. consanguinea Marcelli, Canêz \& Elix, outras espécies brasileiras com sorais orbiculares laminais, não possuem o talo foveolado e o teste $\mathrm{K}$ medular é negativo.

Esta espécie está sendo citada pela primeira vez para o Estado do Maranhão.

Canoparmelia cryptochlorophaea (Hale) Elix \& Hale, Mycotaxon 27: 278. 1986 三 Parmelia cryptochlorophaea Hale, Bryologist 62: 18. 1959. Tipo: República Dominicana, Trujillo, leg. Allard $15715 a$ (holotipo: US), fide Hale (1976).

Distribuição conhecida: Américas e Ásia (Hale 1976). No Brasil foi citada para CE, MG e RJ (Jungbluth 2006).

Descrição e ilustração: Hale (1976), Jungbluth (2006), Zanetti (2014).

Testes de coloração: córtex superior K+ amarelo, UV-; medula $\mathrm{K}-, \mathrm{C}-, \mathrm{KC}+$ rosa, P-, UV-.

Substâncias de importância taxonômica (CCD): atranorina no córtex superior; ácidos caperático e criptoclorofeico na medula.

Material examinado: BRASIL. MARANHÃo: Município de Estreito, Balneário Águas do Cerrado, em tronco de árvore viva, 5-IX-2009, I.P.R. Cunha \& M.A.L. Dias 4; Município de São João do Paraíso, em tronco de árvore viva, 10-X-2009, I.P.R. Cunha, M.P. Marcelli \& M.A.L. Dias 32, 33, 58, 60; Município de São Pedro dos Crentes, em tronco de árvore viva, 22-VII-2009, leg. I.P.R. Cunha \& M.A.L Dias 292.

Os sorais capitados marginais elevados acima do talo prontamente identificam morfologicamente Canoparmelia cryptochlorophaea. Além disso, a presença de ácido criptoclorofeico (medula $\mathrm{C}-, \mathrm{KC}+$ rosa) é única.

Outras espécies sorediadas com reação medular $\mathrm{KC}+$ comuns no Brasil desenvolvem sorais tipicamente laminais ao invés de marginais; além disso, a reação $\mathrm{KC}$ é de outra tonalidade, $\mathrm{KC}+$ vinho fraco evanescente em C. texana (ácido divaricático) e $\mathrm{KC}+$ avermelhado em C. consanguinea (ácidos evérnico e olivetórico).

Awasthi (1976) afirmou que todos os testes de coloração são negativos. Hale (1976) e Zanetti (2014) asseguraram que o teste $\mathrm{K}$ é negativo, mas o resultado do teste KC é rosado. Para Orange et al. (2001), o ácido criptoclorofeico reage $\mathrm{K}+$ rosado, a mesma reação encontrada nos espécimes coletados no cerrado do Estado do Maranhão.

Esta espécie está sendo citada pela primeira vez para o Estado do Maranhão.

Canoparmelia martinicana (Nyl.) Elix \& Hale, in: Elix, Johnston \& Verdon, Mycotaxon 27:278. 1986. $\equiv$ Parmeliamartinicana Nyl., Flora 68: 609. 1885. Tipo: Martinique, Jardin, 1860 (H-Nyl, holotipo).

Figura 1

Distribuição conhecida: Caribe e regiões das Américas Central e do Norte próximas ao Caribe (Hale 1976).

Corticícola, cinza, levemente amarronzado no centro, totalmente opaco, aveludado a $10 \times$, minuto acetinado até $30 \times$, acetinado a $40 \times$, sublobado, ca. $10 \mathrm{~cm}$ de extensão. Lobos subdicotômicos anisotômicos a simpodiais, base 0,3-2,0 mm larg., maior largura 1,6-3,0 mm, contíguos a sobrepostos lateralmente, adnatos, eixo longitudinal distendido, corte transversal plano. Superfície superior firme, contínua na parte distal, se torna rachada a reticulada quando o talo se enruga em direção ao centro, onde cada aréola corresponde a uma ruga ou tubérculo; bastante escrobiculada nas partes mais jovens, passa a longitudinalmente pregueada em alguns lobos e finalmente lisa quando o talo fica mais espesso e antes de se tornar tuberculado e rugoso no centro. Pruína 
ausente. Margem lateral lisa, sinuosa (difícil de ver pela sobreposição dos lobos) e plana, apresenta lobos adventícios nas partes mais velhas ou danificadas; linha negra sutil, presente apenas nas partes mais distais. Zona apical coplanar à lâmina. Ápice crenado a subtruncado, prostrado. Axilas redondas a auriculadas, pequenas (a maior parte difícil de enxergar). Lacínulos concoloridos frequentes entremeados por raros lóbulos; marginais, abundantes na zona proximal, principalmente nas proximidades das axilas e em lobos mais velhos, onde podem também se originar dos tubérculos laminais mais velhos, porém sempre nas proximidades da margem; a maioria simples, os mais longos irregularmente furcados ou ramificados $0,1-1,0 \times 0,1-0,2 \mathrm{~mm}$, o mais curtos lobuloides 0,1-1,0 × 0,1-1,0 mm; planos; coplanares lâmina; ápice arredondado; linha negra ausente; lado de baixo enegrecido e em parte dotados de rizinas; se desenvolvem por toda a margem da zona proximal, concentrados principalmente nas proximidades das axilas e nas partes mais velhas e rugosas do talo, onde são densos e mesmo amontoados; surgem como pequenas projeções marginais ou a partir dos tubérculos laminais mais centrais, achatando-se logo em seguida e crescendo, uns mais circulares (com fixação excêntrica) e outros lacinulares, que logo se ramificam no mesmo padrão dos sublobos do talo. Máculas muito evidentes, reticulares a efiguradas, laminais, e nas cristas dos escrobículos jovens dos lobos mais jovens e mais aderidos ao substrato, menos evidentes em lobos não aderidos e aqueles não ou pouco escrobiculados; todas, porém, logo desaparecendo em direção ao centro, o que torna a maior parte do talo emaculado. Cílios ausentes. Pústulas e sorédios ausentes. Isídios na maioria concoloridos, alguns com a lateral do ápice levemente escurecido, como se fossem desenvolver a coloração do lado de baixo, uma parte deles com ápice expondo a medula branca, opacos como o talo, de frequentes a abundantes, mais adensados em direção ao centro e ausentes da



Figura 1. Canoparmelia martinicana (Nyl.) Elix \& Hale. a. Aspecto geral do talo. b. Detalhe da região distal, máculas evidentes. c. Detalhe da região proximal, isídios pequenos e ramificados (jovens). d. Detalhe dos apotécios dobrados e de margem fendida, regiões decorticadas do anfitécio (manchas brancas). Escalas: $\mathrm{a}=10 \mathrm{~mm}$; b, c, $\mathrm{d}=2 \mathrm{~mm}$.

Figure 1. Canoparmelia martinicana (Nyl.) Elix \& Hale. a. Habitus. b. Detail of the evident maculation in the distal region of the thallus. c. Detail of the proximal region, small ramified (young) isidia. d. Detail of the folded cleft apothecia, amphithecial decorticate portions (white patches). Scales: $a=10 \mathrm{~mm} ; \mathrm{b}, \mathrm{c}, \mathrm{d}=2 \mathrm{~mm}$. 
parte distal escrobiculada; firmes, porém uma parte procumbente; destacam-se facilmente ao toque, mas não há sinais de caducidade; rígidos; laminais; os jovens granulares crescem até irregularmente cilíndricos; retos a levemente sinuosos por causa do processo de proliferação; ápice arredondado; base constrita; ramificados curto-antleriformes com até 3 ramificações; 0,05-0,15 × 0,02-0,03 mm; cílios ausentes; os mais distais nascem laminais, porém principalmente associados a pequeninas elevações da superfície ou às cristas dos escrobículos; na parte proximal a maior parte nasce na parte superior dos tubérculos ou, principalmente, das bordas das quebras do córtex, quando, então, mantêm o ápice com a medula exposta com uma aparência de erodida de feltro; crescem por proliferação, o que torna o isídio evidentemente toruloso. Medula branca, de densidade normal. Lado de baixo: Zona marginal inferior marrom bem clara (casca de noz), subopaca, aveludado a 10×, subacetinado a $20 \times$, ainda minuto acetinado a $30 \times$, acetinado a $40 \times$; largura $(\mathrm{mm})$ 0,5-5,0 $\mathrm{mm}$, porém o limite é muitíssimo atenuado, com uma larga transição em que o marrom escurece lentamente em direção ao centro negro; papilada (lisa entre as papilas); rizinado após as papilas (rizinas jovens originadas das papilas). Parte proximal inferior negra, opaca, o acetinado idêntico à zona marginal; na maior parte contínua, pouco fendida em certos lobos (acidental); densamente rugulosa (rúgulas bem baixas e de orientação variada). Rizinas frequentes a abundantes, homogeneamente distribuídas, presentes inclusive na zona marginal, monomórficas, concoloridas ao lado de baixo, uma parte com o ápice mais claro (principalmente as mais jovens), subopacas; o acetinado idêntico à zona marginal; não ramificadas; retas; as jovens afiladas, as velhas cilíndricas; $0,2 \times<0,01$. Apotécios subcupuliformes, até $5,5 \mathrm{~mm}$ diâm., adnatos, laminais; disco badio epruinoso, na maior parte inteiros, fendidos apenas os mais velhos, com 1-3(-5) fendas pouco profundas $(<1 / 5$ do raio, a maioria involuto desde jovem, o número de dobras dependendo da quantidade de fendas, imperfurado; margem $0,1 \mathrm{~mm}$, lisa, isídios ocasionalmente desenvolvidos (não caracteristicamente marginais do apotécio), margem interna irregularmente crenulada com a idade, também com isídios casuais; anfitécio inicialmente liso, passa a levemente amarrotado e, nos mais velhos, subescrobiculado, quando então as cristas dos escrobículos se tornam decorticadas (a aparência no menor aumento é de máculas, pois são regiões mais claras), emaculado, pouco isidiado e de modo casual (muitos apotécios não tem isídios); estipe ausente; himênio $80 \mathrm{~mm}$ alt.; subhimênio prosoplectenquimático, $20 \mathrm{~mm}$ alt., formado por hifas horizontais de paredes espessas; hipotécio escleroplectenquimático a prosoplectenquimático, de altura irregular, 25-50 mm alt., cartilagíneo, formado por células de paredes muito espessadas mas com lume facilmente visível, porém reunidas em grupos mais ou menos esféricos ou sinuosos bem distintos, não num tecido contínuo. Ascosporo elipsoide reto, $16 \times 8 \mathrm{~mm}$, episporo ca. $1 \mathrm{~mm}$, gútulas ausentes. Picnídios subapicais (poucos encontrados), imersos, ostíolo negro. Conídios não encontrados.

Testes de coloração: córtex $\mathrm{K}+$ amarelo, $\mathrm{P}+$ amarelo; medula $\mathrm{K}+$ amarelo, $\mathrm{C}+$ amarelo fraco, $\mathrm{KC}+$ amarelo $\rightarrow$ alaranjado aguado $\rightarrow$ pardo claro, $\mathrm{P}+$ laranja.

Substâncias de importância taxonômica (CCD): atranorina cortical e ácido protocetrárico medular.

Material examinado: BRASIL. MARANHÃo: Município de Carolina, Fazenda Santa Rita, cerrado, sobre tronco de árvore viva, 22-III-2009, I.P.R. Cunha \& M.A.L. Dias 228-35.

Material adicional: TRINIDAD, Small Bay (local exato ilegível), on bark of a tree, 29-V-1932, leg. W.E. Broadway 8099 (S!) - det. M.E. Hale em 1959.

Canoparmelia martinicana é a única espécie do gênero no Brasil com isídios laminais, portadora de ácido protocetrárico (medula $\mathrm{P}+$ laranja), cujos ramos mais distais são evidentemente escrobiculados e maculados e cujos isídios são em parte decorticados no ápice, características que prontamente a diferenciam de $C$. amazonica (vide comentários sob essa espécie).

Apenas um ascosporo aparentemente normal e maduro foi encontrado após o corte de vários apotécios; um asco com 8 esporos murchos pouco menores foi visto, além de vários sinais de esporos murchos. Talvez a produção de ascósporos seja sazonal na espécie, pois os himênios estão todos no mesmo estágio de maturação, independente do tamanho dos apotécios.

No Brasil, outras espécies isidiadas mais ou menos semelhantes em hábito apresentam máculas por todo o talo ou são emaculadas, têm isídios íntegros e as reações de coloração medulares são bastante diferentes pela presença de outras substâncias medulares, e.g., Parmelinella cinerascens (Lynge) Benatti \& Marcelli e C. salacinifera reagem $\mathrm{K}+$ amarelo para vermelho (ácido salazínico), C. sanguinea Marcelli \& Benatti e C. roseoreagens Marcelli \& Canêz reagem K- e $\mathrm{C}+$ avermelhado (ácidos evérnico e olivetórico), C. caroliniana (Nyl.) Elix \& Hale tem medula K-e 
$\mathrm{C}+$ rosado aquoso evanescente (ácido perlatólico) e C. cassa Marcelli \& C.H. Ribeiro tem a medula K- e C- (sem substâncias medulares reativas).

Esta espécie está sendo citada pela primeira vez para o Brasil, no Estado do Maranhão.

Canoparmelia texana (Tuck.) Elix \& Hale, Mycotaxon 27: 279. 1986. $\equiv$ Parmelia texana Tuck., American Journal of Science and Arts, series 2, 25: 424. 1858. Tipo: U.S.A. Texas, leg. Wright (lectotipo: FH; isolectotipos: M, US), fide Hale (1976).

Distribuição conhecida: continente americano e África (Hale 1976). No Brasil foi citada para MG, MS, PR, RS, SC e SP (Jungbluth 2006).

Descrição e ilustração: Hale (1976), Jungbluth (2006), Zanetti (2014).

Testes de coloração: córtex superior K+ amarelo, UV-; medula $\mathrm{K}-, \mathrm{C}-, \mathrm{KC}+$ rosa pálido, $\mathrm{P}-, \mathrm{UV}+$ branco azulado.

Substâncias de importância taxonômica (CCD): atranorina no córtex superior; ácidos divaricático e nordivaricático na medula.

Material examinado: BRASIL. MARANHÃo: Município de Carolina, Fazenda Santa Rita, em árvore viva, no cerrado, 10-X-2009, I.P.R. Cunha, M.P. Marcelli \& M.A.L. Dias 42, 51 .

Canoparmelia texana, uma das espécies de fungos liquenizados mais frequentes no Brasil, é facilmente reconhecida pelos sorais laminais bem orbiculares, a superfície inferior negra e os testes medulares negativos, exceto pelo $\mathrm{KC}$, que desenvolve uma coloração rosada ou vinosa a violeta muito fraca e fugidia (na gotícula de líquido aplicado, não na medula) devido à presença dos ácidos divaricático e nordivaricático.

Jungbluth (2006) mencionou que espécimes coletados em cerrados paulistas reagem ao $\mathrm{KC}+$ com coloração violeta. No material procedente da região Tocantina a reação $\mathrm{KC}+$ é rosa pálido e rapidamente evanescente.

Canoparmelia consanguinea tem uma morfologia muito semelhante, mas difere de C. texana pela constituição química (ácidos olivetórico, sequicaico e anziaico), que atribui à medula uma forte reação $\mathrm{C}+$ e KC+ avermelhada (Canêz et al. 2009).

Canoparmelia cryptochlorophaea, também é sorediada e a medula reage ao teste $\mathrm{KC}$, porém com uma coloração rosada mais estável na medula (ácido criptoclorofeico) e seus sorais são capitados marginais e elevados.
Crespoa crozalsiana, também apresenta sorais em parte orbiculares, mas tanto a morfologia dos ramos (foveolados) quanto à constituição química (complexo stíctico; medula $\mathrm{K}+, \mathrm{P}+$ amarelo e $\mathrm{KC}$-) são muito diferentes.

Canoparmelia texana é um dos fungos liquenizados mais frequentemente encontrados em zonas urbanas, inclusive com ar bastante poluído (Marcelli 1998, Saiki et al. 2001), e em formações vegetais abertas (e.g., cerrado) por todo o sul e sudeste do Brasil e, por isso, obviamente esperada em grande quantidade na região tocantina. A presença de apenas um indivíduo pequeno em toda a amostragem foi algo notável e inesperado.

Parmelinella salacinifera (Hale) Marcelli \& Benatti, in Benatti, Mycosphere 5: 770-789. 2014. $\equiv$ Canoparmelia salacinifera (Hale) Elix \& Hale, Mycotaxon 27:279. 1986. $\equiv$ Parmelia salacinifera Hale, in Hale \& Kurokawa, Contrib. U.S. Nat. Herb. 36: 157. 1964. Tipo: U.S.A., Florida, Sanford, Seminole County, leg. Rapp, (holotipo: US; isotipo: FLAS), fide Hale (1976).

Distribuição conhecida: continente americano e Ásia (Hale 1976). No Brasil foi citada para MA, MT e SP (Jungbluth, 2006)

Descrição e ilustração: Hale (1976) e Jungbluth (2006), respectivamente como Pseudoparmelia salacinifera e Canoparmelia salacinifera.

Testes de coloração: córtex superior $\mathrm{K}+$ amarelo, $\mathrm{UV}$-; medula $\mathrm{K}+$ amarelo $\rightarrow$ vermelho, $\mathrm{C}-, \mathrm{KC}-\mathrm{P}+$ amarelo, UV-.

Substâncias de importância taxonômica (CCD): atranorina no córtex superior; ácidos consalazínico e salazínico na medula.

Material examinado: BRASIL. MARANHÃO: município de Carolina, Fazenda Santa Rita, em tronco de árvore viva, 22-III-2009, I.P.R. Cunha \& M.A.L. Dias 23/58; idem, 6-IX-2009, I.P.R. Cunha, J. Bordin \& M.A.L. Dias 10940, 1866; idem, 10-X-2009, I.P.R. Cunha, M.P. Marcelli \& M.A.L. Dias 974, 27131, 37141; idem, Município de São Pedro dos Crentes, 23-VII-2009, I.P.R. Cunha \& M.A.L. Dias 256, 271; idem, Município São João do Paraíso, em tronco de árvore viva, 10-X-2009, I.P.R. Cunha, M.P. Marcelli \& M.A.L. Dias 11. Tocantins: Município de Itaguatins, Fazenda São Paulo, 1-I-2009, I.P.R. Cunha \& M.A.L. Dias 4, 14, 48; idem, Município de São Bento do Tocantins, 9-IX-2009, I.P.R. Cunha, J. Bordin \& M.A.L. Dias 18, 24. 
Parmelinella salacinifera, recentemente transferida do gênero Canoparmelia (Benatti, 2014) é uma espécie emaculada, com isídios laminais, prontamente reconhecida no Brasil pela superfície inferior castanha clara e presença dos ácidos consalazínico e salazínico na medula $(\mathrm{K}+$ amarelo para vermelho).

Parmelinella cinerascens, cujo talo e isídios são bastante semelhantes em hábito e também produz ácido salazínico, tem a superfície inferior negra (Benatti 2012).

Canoparmelia amazonica e C. cassa, emaculadas, possuem reações medulares diferentes e superfície inferior negra. Canoparmelia caroliniana pode apresentar córtex inferior castanho, mas apresenta ácido perlatólico na medula $(\mathrm{KC}+$ violeta, $\mathrm{UV}+$ branco azulado) ao invés de ácido consalazínico e salazínico e é fortemente maculada (Jungbluth 2006, Zanetti 2014).

\section{Agradecimentos}

I.P.R. Cunha agradece a UNISULMA (Unidade de Ensino Superior do Sul do Maranhão), pela disponibilidade do laboratório de Bioquímica e Biologia e pela bolsa concedida. E.C. Pereira e M.P. Marcelli, agradecem ao CNPq por bolsa de Produtividade em Pesquisa.

\section{Literatura citada}

Benatti, M.N. 2012. Canoparmelia cinerascens belongs in the genus Parmelinella (Parmeliaceae, lichenized Ascomycota). Opuscula Philolichenum 11: 26-30.

Benatti, M.N. 2014. An update on the genus Parmelinella Elix \& Hale (Parmeliaceae, lichenized ascomycetes). Mycosphere 5: 770-789.

Brodo, I.M., Sharnoff, S.D. \& Sharnoff, S. 2001. Lichens of North America. Yale University Press, New Haven e London.

Canêz, L. \& Marcelli, M.P. 2006. Gêneros de Parmeliaceae (Ascomycetes Liquenizados) na localidade de Fazenda da Estrela, Vacaria, Rio Grande do Sul, Brasil. Caderno de Pesquisa, Série Biologia, 18: 38-81.

Canêz, L., Marcelli, M.P. \& Elix, J.A. 2009. New Brazilian species of Canoparmelia with medullary olivetoric, anziaic, and sekikaic complexes. Mycotaxon 110: 465-472.

Elix, J.A. 1994. Parmeliaceae. Flora of Australia 55: 1-360.

Elix, J.A. 2014. ACatalogue of Standardized Chromatographic Data and Biosynthetic Relationships for Lichen Substances. 3 ed. Published by the author, Canberra.
Elix, J.A., Johnston, J. \& Verdon, D. 1986. Canoparmelia, Paraparmelia and Relicinopsis, three new genera in the Parmeliaceae (lichenized Ascomycotina). Mycotaxon 27: 271-282.

Hale, M.E. 1976. A monograph of the lichen genus Pseudoparmelia Lynge (Parmeliaceae). Smithsonian Contributions to Botany 31: 1-62.

Hale, M.E. 1979. How to know the Lichens. WM. \& C. Brown Company Publishers. Dubuque.

Huneck, S. \& Yoshimura, I. 1996. Identification of lichen substances. Springer, Berlin.

Jungbluth, P. 2006. A família Parmeliaceae (fungos liquenizados) em fragmentos de cerrados do Estado de São Paulo. Dissertação de Mestrado, Instituto de Botânica, São Paulo.

Lendemer, J.C. \& Hodkinson, B.P. 2012. Recognition of the Parmelia crozalsiana group genus Crespoa. North American Fungi. 7: 1-5.

Marcelli, M.P. 1998. History and current knowledge of Brazilian lichenology. In: Marcelli, M.P. \& Seaward, M.R.D. (eds.). Lichenology in Latin America: history, current knowledge and applications, pp. 25-45. CETESB, São Paulo.

Martins, M.B \& Oliveira, T.G. 2011. Amazônia Maranhense: diversidade e conservação. MPEG, Belém.

Nash III, T.H. \& Elix, J.A. 2002. Canoparmelia. In: Nash III, T.H., Ryan, B.D., Gries, C. \& Bungartz, F. (eds.). Lichen Flora of the greater Sonoran Desert Region, v. 1, pp. 122-125. Arizona State University, Tempe.

Nylander, W. 1885. Parmeliae exoticae novae. Flora 68: 605-616.

Orange, A. James, P.W. \& White, F.J. 2001. Microchemical methods for the identification of lichens. British Lichen Society. London.

Saiki, M., Horimoto, L.K., Vasconcellos, M.B.A., Marcelli, M.P. \& Coccaro, D.M.B. 2001. Survey of elemental concentrations in lichen samples collected from São Paulo State. Journal of Radioanalytical and Nuclear Chemistry 249: 317-320.

Sousa, J.M. 2009. A cidade na região e a região na cidade - a dinâmica socioeconômica de Imperatriz e suas implicações na região Tocantina. Editora Ética, Imperatriz.

TOCANTINS. 2011. Tocantins. Disponível em http:// to.gov.br/tocantins/2 (III-2011).

Walker, J.W. \& James, P.W. 1980. A revised guide to microchemical techniques for the identification of lichen products. Bulletin of the British Lichen Society 46 (supl.): 13-29.

Zanetti, C.A. 2014. Estudo taxonômico e anatômico em espécies de Canoparmelia s.l. (Parmeliaceae, Ascomycota liquenizados). Dissertação de Mestrado, Universidade Estadual Paulista, Botucatu. 